Journal of Computational and Applied Mechanics, Vol. 11., No. 2., (2016), pp. 123-136 DOI: $10.32973 /$ jcam.2016.009

\title{
NUMERICAL MODELING OF A DIRECT SPRING OPERATED PRESSURE RELIEF VALVE
}

\author{
ISTVÁN ERDŐDI \\ Budapest University of Technology and Economics \\ 1111 Müegyetem rkp. 3., Budapest, Hungary \\ ierdodi@hds.bme.hu \\ CsABA HöS \\ Budapest University of Technology and Economics \\ 1111 Müegyetem rkp. 3., Budapest, Hungary \\ cshos@hds.bme.hu
}

[Received: March 25, 2016, Accepted: July 22, 2016]

\begin{abstract}
This paper presents two methods for modeling the response of a direct spring operated pressure relief valve: one approach uses one-dimensional gas dynamical equations coupled with the equation of motion of a one degree-of-freedom oscillator, while the other employs deforming mesh CFD simulations to fully resolve the flow field. We found that if the force and flow rate characteristics of the valve are implemented into the reduced order model, it yields approximately the same results as the CFD computations.
\end{abstract}

Mathematical Subject Classification: 76N15, 76-04

Keywords: CFD, linear stability, nonlinear vibrations, pressure relief valve

\section{INTRODUCTION}

Pressure relief valves (PRVs) are used as the last line of defense against overpressure in industrial environments. There are two main parameters of these valves regarding their operation: the set pressure $\left(p_{\text {set }}\right)$, which is the minimum pressure at which the valve opens, and the capacity, that is by definition the flow rate through the valve at full lift and at $110 \%$ of the set pressure. The latter describes how fast the overpressure can be reduced to the desired level and maintaining full capacity is critical from a safety point of view. It is possible that under certain circumstances instabilities arise, which reduce the capacity, endangering the whole system. The goal of this paper is to investigate the behavior of a direct spring operated PRV in gas service with a focus on stability. This configuration consists of a disc pressed against the seat at the pipe end by a pre-compressed spring. The advantage of this simple design is that the set pressure can be easily adjusted through the pre-compression of the spring, and the probability of mechanical failure can be kept at a minimal level due to the low number of moving parts. In order to avoid exceeding the time available for blowdown, adding any kind of artificial damping is forbidden by the current industrial standards [1. 
Based on this, the valve disc can be modeled as a one degree-of-freedom oscillator, which - due to the force acting on it - is described by a non-linear differential equation. This means that dynamic instabilities must be taken into account. The RP520 standard of the American Petroleum Institute distinguishes three different kinds of instabilities for direct spring operated PRVs. The first is the so-called cycling, during which the set pressure slowly builds up after the valve closes, resulting in small frequency $(<1 \mathrm{~Hz})$ vibrations. Contrary to this, both flutter and chatter are large frequency $(>10 \mathrm{~Hz})$ self-excited vibrations - the difference between them is that during chatter the amplitude is so large that the disc usually impinges on the seat and upper stopper, resulting in not only a decrease in capacity but mechanical damage as well. Cycling is well-understood and means to avoid it are already included in the standards. However, the reasons behind flutter and chatter are not completely clear according to API, but it is mentioned that these are due to the acoustic coupling of the valve disc and the pipe end. Ongoing research activities aim to gain a better understanding of these phenomena 2, 3. The goals of this paper are (a) to provide a steady-state CFD model for the evaluation of the force acting on the valve disc and the capacity, (b) to study the transient valve response by a deforming mesh CFD model and (c) to compare the results of unsteady CFD runs against a simplified ODE (ordinary differential equation) model including quasi-steady fluid force characteristics.

\section{Theoretical BaCkground}

The system under analysis consists of a straight pipe section and the valve itself. The governing equations describing the behavior of these components are derived in this section.

2.1. The valve. As stated before, the valve disc can be modeled as an oscillator:

$$
m \ddot{x}+k \dot{x}+s\left(x+x_{0}\right)=F_{\text {total }},
$$

where $m$ is the reduced mass of the system, $x$ is the valve lift, $k$ is the damping coefficient, $s$ is the spring stiffness, $x_{0}$ is the pre-compression of the spring and $F_{\text {total }}$ is the total force acting on the valve. The force can be traced back to three physical phenomena: force acts because of the pressure difference between the upstream and downstream pressures, due to the change in the momentum of the out-flowing gas, and originates from viscous effects, i.e.

$$
F_{\text {total }}=F_{\text {pres }}+F_{\text {mom }}+F_{\text {visc }}
$$

where the force from the pressure distribution is

$$
F_{\text {pres }}=A_{\text {pipe }}\left(p_{\mathrm{v}}-p_{\mathrm{b}}\right),
$$

where $A_{\text {pipe }}$ is the cross-sectional area of the pipe, $p_{\mathrm{v}}$ is the pressure upstream of the valve and $p_{\mathrm{b}}$ is the back pressure. The problem is that $F_{\text {mom }}$ and $F_{\text {visc }}$ cannot be calculated analytically, as neither the velocity nor the direction of the flow are known. Their effects can be taken into account through the effective area, which supposes that 
the total force can be evaluated as a multiplication of the static pressure difference and an area function [2], that is

$$
F_{\text {total }}\left(x, p_{v}\right)=A_{\text {eff }}(x)\left(p_{\mathrm{v}}-p_{\mathrm{b}}\right),
$$

where $A_{\text {eff }}$ is the effective area function. As there is no flow when the valve is seated, i.e. $x=0$, the effective area for that position equals the cross-sectional area of the pipe, resulting in

$$
A_{\text {eff }}(0)=A_{\text {pipe }}
$$

Substituting the effective area into Equation (2.1) gives

$$
m \ddot{x}+k \dot{x}+s\left(x+x_{0}\right)=A_{\text {eff }}(x)\left(p_{\mathrm{v}}-p_{\mathrm{b}}\right) .
$$

It can be seen that this equation is indeed a non-linear ordinary differential equation. One of the goals of the stationary mesh CFD simulations presented later is to obtain the $A_{\text {eff }}(x)$ function for the given PRV.

2.2. Stability of the linearized system. The first step in the linear stability analysis is finding the equilibria $x_{\text {eq }}$ from equation (2.6), taking into account that $\dot{x}_{\text {eq }} \equiv 0$ and $\ddot{x}_{\text {eq }} \equiv 0$, i.e.

$$
x_{\mathrm{eq}}=\frac{A_{\mathrm{eff}}\left(x_{\mathrm{eq}}\right)}{s}\left(p_{\mathrm{v}, \mathrm{eq}}-p_{\mathrm{b}}\right)-x_{0} .
$$

Linearizing the effective area around this point results in

$$
A_{\text {eff }}(x) \approx A_{\text {eff }}\left(x_{\mathrm{eq}}\right)+\left.\frac{\mathrm{d} A_{\mathrm{eff}}(x)}{\mathrm{d} x}\right|_{x=x_{\mathrm{eq}}}\left(x-x_{\mathrm{eq}}\right),
$$

which can be substituted back into Equation 2.6 to get

$$
m \ddot{x}+k \dot{x}+s\left[\left(x-x_{\mathrm{eq}}\right)+x_{\mathrm{eq}}+x_{0}\right] \approx\left(A_{\mathrm{eff}}\left(x_{\mathrm{eq}}\right)+\left.\frac{\mathrm{d} A_{\mathrm{eff}}(x)}{\mathrm{d} x}\right|_{x=x_{\mathrm{eq}}}\left(x-x_{\mathrm{eq}}\right)\right)\left(p_{\mathrm{v}}-p_{\mathrm{b}}\right) .
$$

Utilizing that $x_{\mathrm{eq}}$ is an equilibrium solution and introducing $\zeta=x-x_{\text {eq }}$ gives

$$
m \ddot{\zeta}+k \dot{\zeta}+\left(s-\left.\frac{\mathrm{d} A_{\mathrm{eff}}}{\mathrm{d} x}\right|_{x=x_{\mathrm{eq}}}\left(p_{\mathrm{v}}-p_{\mathrm{b}}\right)\right) \zeta=0 .
$$

This differential equation is linear, and the role of the effective area is highlighted: the linearised force decreases the coefficient of the displacement, i.e. the spring stiffness. The term in the brackets can be regarded as an "effective spring stiffness", and loss of stability occurs when it becomes negative, i.e.

$$
s-\left.\frac{\mathrm{d} A_{\mathrm{eff}}}{\mathrm{d} x}\right|_{x=x_{\mathrm{eq}}}\left(p_{\mathrm{v}}-p_{\mathrm{b}}\right)<0 .
$$

Therefore, the stability of an equilibrium solution for a given pressure difference and spring stiffness can be determined from the derivative of the effective area at the equilibrium displacement, meaning that knowing the $A_{\text {eff }}(x)$ function is crucial from the point of stability as well. 
2.3. Mass flow rate through the valve. As stated before, the mass flow rate through the valve is one of the most important parameters. Two cases must be distinguished based on the ratio of the pressure at the pipe end and the back pressure. The flow is said to be choked if this ratio is above the critical pressure ratio [4, which for air $(\gamma=1.4)$ is

$$
\left(\frac{p_{\mathrm{v}}}{p_{\mathrm{b}}}\right)_{\text {critical }}=\left(\frac{\gamma+1}{2}\right)^{\frac{\gamma}{\gamma-1}}=1.8929
$$

and the formula for the mass flow rate 4 is

$$
\dot{m}_{\text {out }}=C_{\mathrm{D}} A_{\text {ref }} \sqrt{\gamma \rho_{\mathrm{v}} p_{\mathrm{v}}\left(\frac{2}{\gamma-1}\right)^{\frac{\gamma+1}{\gamma-1}}},
$$

where $\rho_{\mathrm{v}}$ is the density upstream of the valve and the reference area equals

$$
A_{\text {ref }}=D_{\text {pipe }} \pi x \text {. }
$$

The most problematic part of Equation 2.13 is the discharge coefficient $C_{\mathrm{D}}$ - the other main goal of the stationary mesh CFD simulations is to investigate its values at various valve lifts and pipe end pressures. Similarly, the equation for the mass flow rate for compressible non-choked flows [5] is

$$
\dot{m}_{\text {out }}=C_{\mathrm{D}} A_{\text {ref }} \sqrt{2 \rho_{\mathrm{v}} p_{\mathrm{v}}\left(\frac{\gamma}{\gamma-1}\right)\left[\left(\frac{p_{\mathrm{b}}}{p_{\mathrm{v}}}\right)^{\frac{2}{\gamma}}-\left(\frac{p_{\mathrm{b}}}{p_{\mathrm{v}}}\right)^{\frac{\gamma+1}{\gamma}}\right]} .
$$

2.4. Interaction between the valve and the seat. There is also the possibility of the valve disc hitting the end of the pipe, which phenomenon is not covered by the equations listed above. This event can have two outcomes: either the disc bounces back with a set loss of kinetic energy or it sticks to the pipe end. As these are not covered by the deforming mesh simulations, their modelling can be omitted if the sole goal of the $1 \mathrm{D}$ model is to produce a comparable output to the CFD results.

2.5. The pipe. The flow in the pipe can be regarded as a one-dimensional, unsteady, subsonic, compressible gas flow with wall friction, which is described by the following system of partial differential equations [6. The continuity is

$$
\frac{\partial \rho}{\partial t}+\frac{\partial \rho v}{\partial \xi}=0
$$

the $1 \mathrm{D}$ equation of motion with pipe friction looks like

$$
\frac{\partial \rho v}{\partial t}+\frac{\partial\left(\rho v^{2}+p\right)}{\partial \xi}=\frac{\lambda(v) \rho}{2 D_{\text {pipe }}} v|v|
$$

and the adiabatic energy equation is

$$
\frac{\partial \rho e}{\partial t}+\frac{\partial(\rho v e+p v)}{\partial \xi}=0
$$

where $p$ is the pressure, $v$ is the velocity, $e$ is the specific energy, $t$ is the time, $\xi$ is the spatial coordinate along the pipe, $\lambda$ is the Darcy friction factor and $D_{\text {pipe }}$ is the 
diameter of the pipe. The friction factor was defined as a function of the velocity using Blasius' approximation for turbulent flows [7, which is

$$
\lambda(v)=0.316(\operatorname{Re}(v))^{-0.25}=0.316\left(\frac{v D_{\text {pipe }}}{\nu}\right)^{-0.25}
$$

2.6. Numerical solution of the system. All of these equations were implemented in the 1D model. Equation (2.6) was solved using a 4th/5th order Runge-Kutte solver, while the Lax-Wendroff method [8] was applied to Equations 2.16) to 2.18). A constant total pressure reservoir boundary condition was defined for one end of the pipe, while the other was coupled with the valve. The compressible method of characteristics was used for the numerical implementation of both [6].

\section{Steady-State CFD Simulations}

The goal of the steady-state CFD simulations is to investigate the flow properties at various fixed lifts and reservoir pressures. With these information it will also be possible to obtain the $A_{\text {eff }}(x)$ and $C_{\mathrm{D}}$ values required for the $1 \mathrm{D}$ model. However, it must be noted that this method of approach assumes that even though these parameters refer to dynamic processes, they can be approximated from steady-state solutions. All the CFD simulations were done using ANSYS-CFX.

Both [9] and [10] investigate the general flow parameters of valves, while [11] uses an approach similar to this work, as it also approximates the dynamic properties from results of fixed lift solutions. An advanced version of this method can be seen in [12]: the authors generated individual meshes for a large number of lifts, and their solver chooses from them based on the calculated displacement values. Its advantage is that the process more or less retains its dynamic nature; however, the valve lifts can only be discrete values and the resolution depends on the number of prepared meshes.

The axisymmetry of both the geometry and the boundary conditions were taken into account, therefore only a wedge shaped domain was generated with a central angle of $5^{\circ}$. This also means that all of the results had to be rescaled for the full $360^{\circ}$ geometry. The dimensions of the modeled valve correspond to the measuring equipment located in the laboratory of the Department of Hydrodynamic Systems in order to ease the comparison of the calculated and the measured results in the future. This includes a pipe with an inner diameter of $40.2 \mathrm{~mm}$ (corresponding to $11 / 2^{\prime \prime}$ nominal) and a valve disc diameter of $55.7 \mathrm{~mm}$. The length of the pipe was set to $10 D_{\text {pipe. }}$

3.1. Simulation settings. Due to the axisymmetry, a wedge shaped domain with one cell in the radial direction was sufficient for the modeling of the problem. The block structured mesh was created using ICEM and consists of 90,787 nodes. The domain, the boundary conditions and the element numbers are illustrated in Figure 1. The thinner lines denote the edges of the blocks. A mesh dependency study was also carried out, which is presented at the end of this section. 


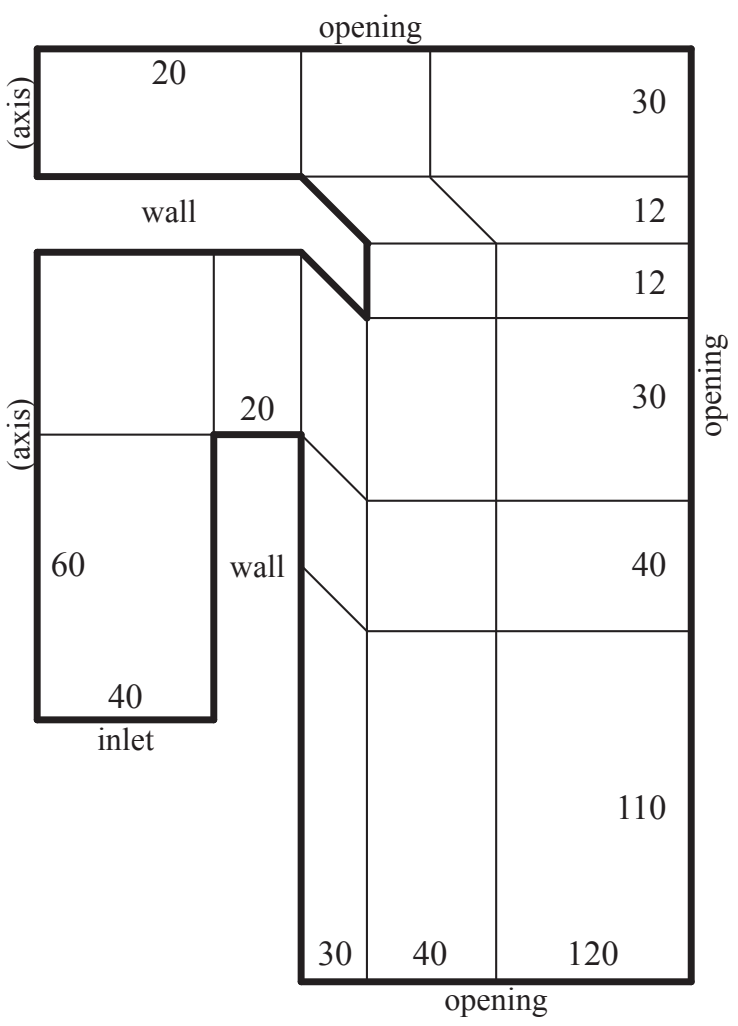

Figure 1. The domain, the boundary conditions and the element numbers (not a proportional illustration)

The opening pressure was set to 1 bar (absolute), while the temperatures at both the inlet and the opening were $293 \mathrm{~K}$. The inlet total pressure was varied between $1-6$ bar and the valve lift range of $0.01-0.35 D_{\text {pipe }}$ was resolved. To ease the notation, relative values for the lift will be used from now on with

$$
\bar{x}=x / D_{\text {pipe }} .
$$

As the steady-state solver only produced converging residuals at very low pressure ratios, a transient solver was used in most cases. These simulations were stopped after both the force acting on the valve and the inlet mass flow rate had converged. The medium was set to air with the ideal gas assumption. High Resolution and Second Order Backward Euler schemes were used for the advection and the time stepping, respectively. The $\mathrm{k}-\varepsilon$ model was chosen for turbulence, because its scalable wall function supposedly provides reasonable accuracy for the boundary layer even without its full numerical resolution [13. A turbulence model dependency study was also concluded, which is presented at the end of this section. The time step is variable and is automatically adjusted by the solver to maintain a set number of inner loop iterations (between 5 and 9). 


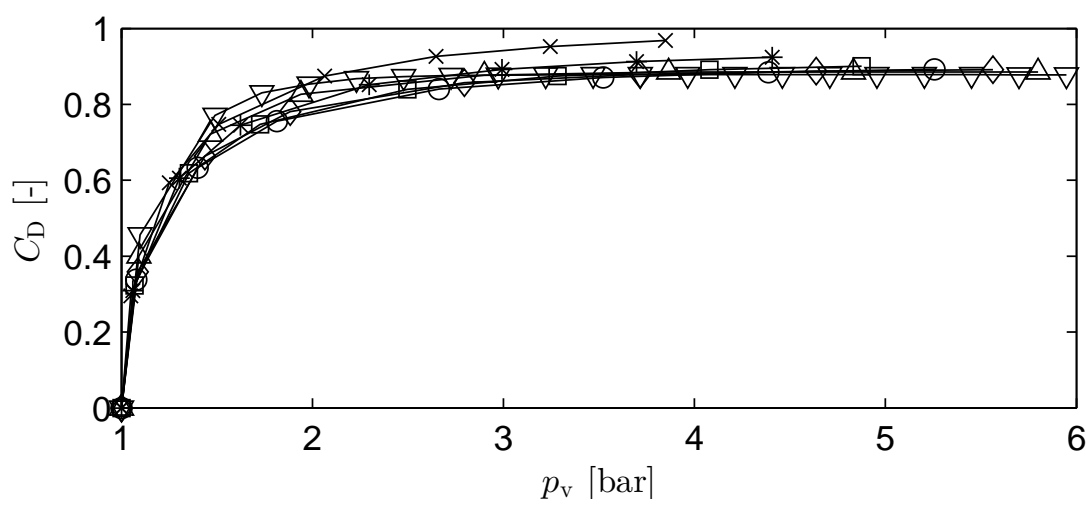

Figure 2. The discharge coefficients at various lifts $(\nabla: \bar{x}=0.05, \triangle$ : $\bar{x}=0.10, \forall: \bar{x}=0.15, \diamond: \bar{x}=0.20, \boxminus: \bar{x}=0.25, *: \bar{x}=0.30, *:$ $\bar{x}=0.35)$

3.2. Results for the discharge coefficient. The discharge coefficients are obtained from Equation 2.13 by substituting the mass flow rates from the CFD simulations $\left(\dot{m}_{\text {out }}=\dot{m}_{\text {out }, \mathrm{CFD}}\right)$, resulting in

$$
C_{\mathrm{D}}=\frac{\dot{m}_{\mathrm{out}, \mathrm{CFD}}}{A_{\mathrm{ref}} \sqrt{\gamma \rho_{\mathrm{v}} p_{\mathrm{v}, \mathrm{CFD}}\left(\frac{2}{\gamma-1}\right)^{\frac{\gamma+1}{\gamma-1}}}} .
$$

The pipe end pressures were also taken from the CFD simulations, while the densities were calculated from the inlet temperature, as experience showed that the variations in density along the pipe are negligible. The discharge coefficients can be seen in Figure 2 Note that the above formula for the mass flow rate is only valid for choked flows, i.e. when the pressure ratio at the valve is above critical, therefore it is not expected to be constant in the non-choked range. After averaging the results in the choked range, a value of $C_{\mathrm{D}}=0.8778$ was obtained for the discharge coefficient.

3.3. Results for the effective area. Equation (2.4) gives the effective areas by substituting the total force acting on the valve and the static pressure from the results of the CFD simulations, that is

$$
A_{\mathrm{eff}}=\frac{F_{\mathrm{total}, \mathrm{CFD}}}{p_{\mathrm{v}, \mathrm{CFD}}-p_{\mathrm{b}}} .
$$

The back pressure $\left(p_{\mathrm{b}}\right)$ is known from the boundary condition, therefore the effective area can be obtained. The ratio of the effective area and the cross-sectional area of the pipe is shown in Figure 3. It confirms that it is reasonable to assume that the effective area depends only on the lift, as it does not significantly vary with the pressure. The $A_{\text {eff }}(x)$ function can be calculated by averaging the values corresponding to the same lifts, which can be seen in Figure 4. It also includes the piecewise cubic interpolation of the function. 


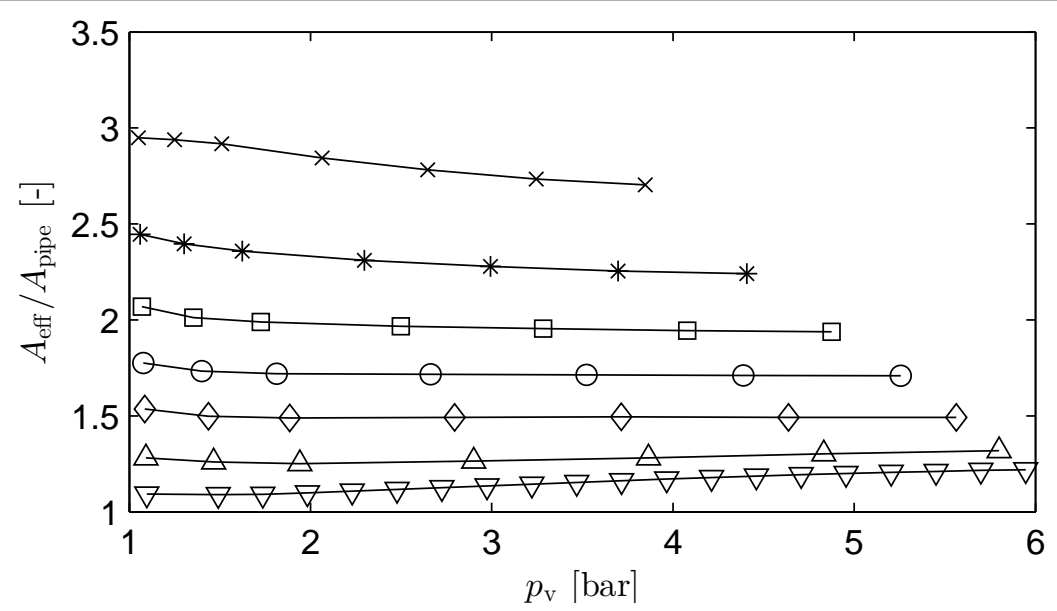

Figure 3. The ratio of the effective area and the cross-sectional area of the pipe at various lifts $(\nabla: \bar{x}=0.05, \triangle: \bar{x}=0.10, \vartheta: \bar{x}=0.15$, ๑: $\bar{x}=0.20, \square: \bar{x}=0.25, *: \bar{x}=0.30, *: \bar{x}=0.35)$

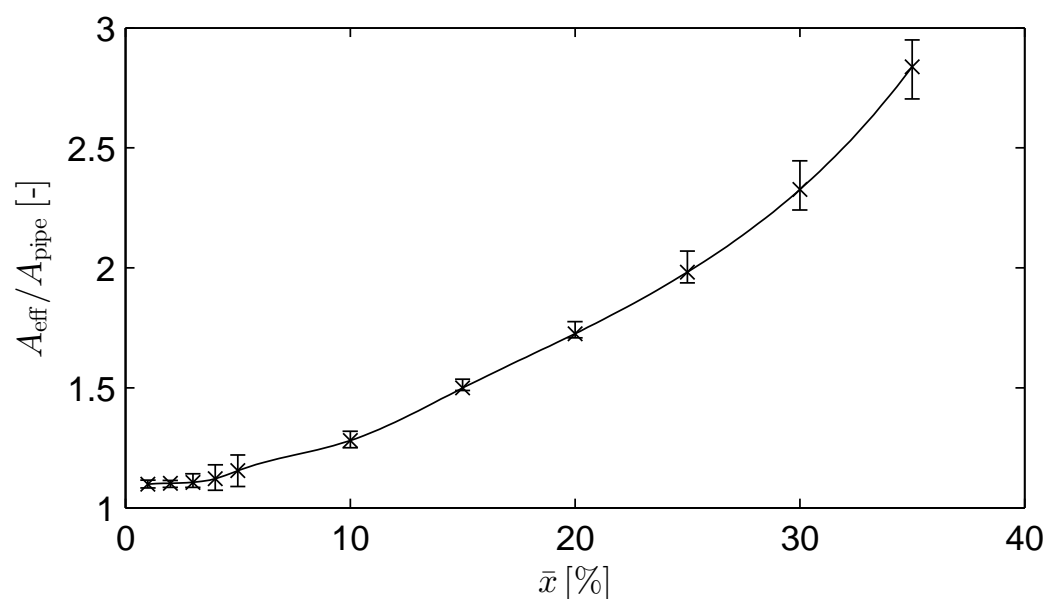

Figure 4. The mean effective area versus the lift $(\times$ : CFD results, -: piecewise cubic interpolation) with the maximum and minimum values (errorbars)

3.4. Equilibrium and stability. First, the equilibrium points must be calculated from Equation (2.7). The pre-compression can be expressed from the set pressure by substituting $x_{\text {eq }}=0$ and utilizing that $A_{\text {eff }}(0)=A_{\text {pipe }}$ :

$$
x_{0}=\frac{A_{\text {pipe }}\left(p_{\text {set }}-p_{\mathrm{b}}\right)}{s}
$$




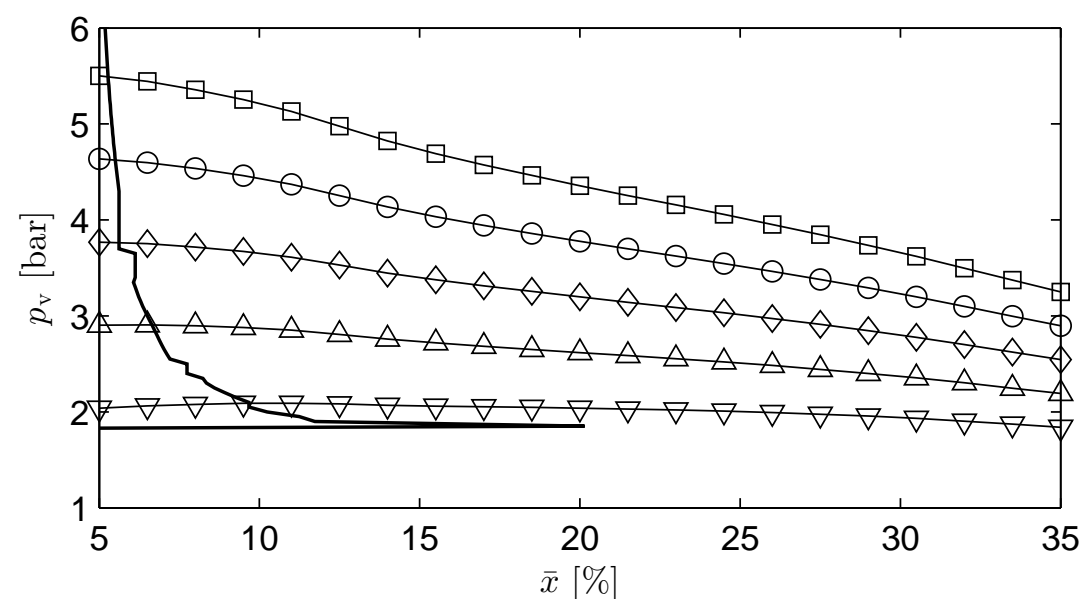

Figure 5. The equilibrium curves $\left(\forall: p_{\text {set }}=2\right.$ bar, $\triangle: p_{\text {set }}=3$ bar, $\diamond: p_{\text {set }}=4$ bar, $\ominus: p_{\text {set }}=5$ bar, $\boxminus: p_{\text {set }}=6$ bar) and the b.o.s. ( $)$

The characteristic curves, i.e. the $p_{\mathrm{v}, \mathrm{eq}}\left(x_{\mathrm{eq}}\right)$ functions corresponding to various set pressures can be formulated using Equations (2.7) and (3.4). The stability of the points on the $p_{\mathrm{v}}-x$ plane was obtained from Equation (2.11). The equilibrium curves for different set pressures and the boundary of stability (b.o.s.) are shown in Figure 5. The equilibria to the left of the b.o.s. are stable. All of these calculations were made with a spring stiffness of $s=12500 \mathrm{~N} / \mathrm{m}$.

3.5. Mesh and turbulence model dependency study. To reduce computational time, both the mesh and the turbulence model dependency studies were only concluded at around the corner points of the investigated inlet total pressure and valve lift domain, i.e. at $p_{\mathrm{t}, \min }=1.5 \mathrm{bar}$ and $p_{\mathrm{t}, \max }=6.0 \mathrm{bar}$, and at $x_{\min }=0.05 D_{\text {pipe }}$ and $x_{\max }=0.35 D_{\text {pipe. }}$. For the mesh dependency study a mesh was generated with two times as many elements on all edges. The relative differences in both the forces acting on the valve and the inlet mass flow rates were under $0.5 \%$, indicating that the domain had been sufficiently resolved by the original mesh. The effect of turbulence modelling was investigated by repeating the simulations in the aforementioned corner points with both the baseline $\mathrm{k}-\omega$ and the k- $\omega$ SST models, and their results were compared to those from the original simulations, where $\mathrm{k}-\varepsilon$ had been used. The largest relative differences were $3.67 \%$ for the force and $5.40 \%$ for the mass flow rate, both of which are sufficiently small.

\section{Deforming Mesh CFD Simulations}

During the calculations of the previous section a method of determining the equilibria and their stability for a direct spring operated pressure relief valve was shown, but it involved the major assumption that the total force acting on the valve can be accurately approximated from steady-state fixed valve results. To investigate the 
effect of unsteadiness, another CFD model was assembled in which the valve disc is free to move in the direction of the pipe axis. The spring is taken into account through the Rigid Body option of CFX with its built-in spring feature. Its stiffness and the reduced mass of the moving bodies had to be defined. As the simulated domain has a centre angle of $5^{\circ}$ (instead of the full $360^{\circ}$ ), both of them had to be rescaled appropriately, i.e. multiplied by $5^{\circ} / 360^{\circ}$. The unloaded position of the spring must also be set, which can be calculated from the pre-compression and the initial position. The movement of the valve disc results in a change in the fluid domain, which is handled by allowing the deformation of the mesh. The Mesh Stiffness was set in a way that the larger the cell volume, the less it deforms in order to preserve the mesh quality at the critical regions (at the walls, in the free jet) as much as possible. The chosen value for the stiffness was

$$
s_{\text {mesh }}=\frac{1}{V_{\text {cell }}}\left[\frac{\mathrm{m}^{2}}{\mathrm{~s}}\right] .
$$

Even though this setting permits large displacements from the initial position of the valve, experience showed that the mesh still suffers a significant loss of quality, to the point that sometimes negative cell volumes would occur. To prevent this, the ICEM CFD replay script based remeshing feature was used, which is covered in detail in [14], only its basics will be summarized here. First of all, a condition for the remeshing has to be defined through an Interrupt Condition - when this expression becomes true, the solver stops. In these simulations it happens when the area average of the Total Mesh Displacement at the valve reaches $1 \mathrm{~mm}$. It proved to be a good quantity to monitor, as its value resets to zero after every remeshing. The remeshing itself is set in a Configuration, which is triggered by the Interrupt Condition. Its ICEM CFD Replay option requires the geometry file (*.tin) of the initial position, a replay script for the meshing (*.rpl), the mapping of the moving part between ICEM and CFX, and a monitor for the total displacement. The area average of the Total Centroid Displacement at the valve was used for the latter (it is not reset during the remeshing). The unit conversion between ICEM and CFX (i.e. from millimeters to meters in our case) is handled by the ICEM CFD Geometry Scale, which was set to 0.001. A remeshing step goes as follows:

1. The Interrupt Condition is fulfilled.

2. The solver stops and ICEM is called.

3. The geometry file is loaded into ICEM and is shifted with the value of the corresponding monitor.

4. The meshing replay script is run on the modified geometry.

5. A new simulation - with the new mesh - is initialized with the results of the stopped one.

This way the required mesh quality can be maintained even for extremely large deformations, as long as no change occurs in the topology. An example for the latter would be the seating of the valve, which is not covered by this model. A similar approach, albeit without remeshing, can be found in [15], but in their case the reservoir was also implemented in the CFD model. 


\section{Comparison of Valve Responses}

To check whether the steady-state assumption of the effective area was correct, 1D simulations based on the model described in the first section were compared to the results of the deforming mesh CFD method. The spring stiffness was set to $12500 \mathrm{~N} / \mathrm{m}$, and its pre-compression was defined such that the valve was in an equilibrium position at the start. The discharge coefficient was also set according to the steady-state simulations $\left(C_{\mathrm{D}}=0.8778\right)$, while the damping was neglected $(k=0)$. Two cases were simulated with the same inlet total pressure jump but with different jump durations. The valve responses and the pressure profiles are illustrated in Figure 6. The correspondence is satisfactory, as the displacement from the $1 \mathrm{D}$ model closely follows that of the deforming mesh solution, even though some difference can be observed in the new equilibria, and also the response seems to be significantly delayed in the case of the slower pressure jump.
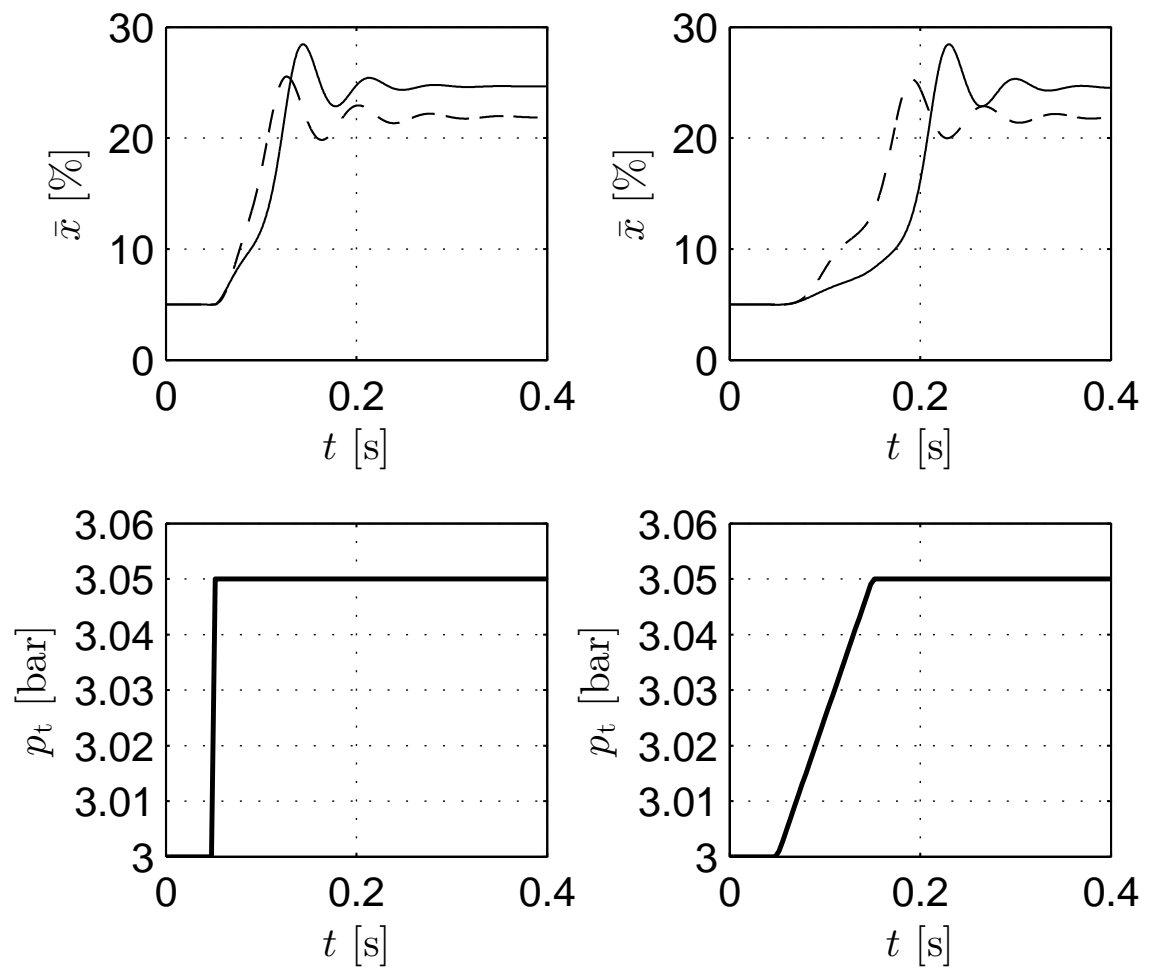

Figure 6. Valve responses of the deforming mesh CFD (cont. line) and the 1D (dashed line) models in the case of a sudden (left) and slow (right) pressure jumps 

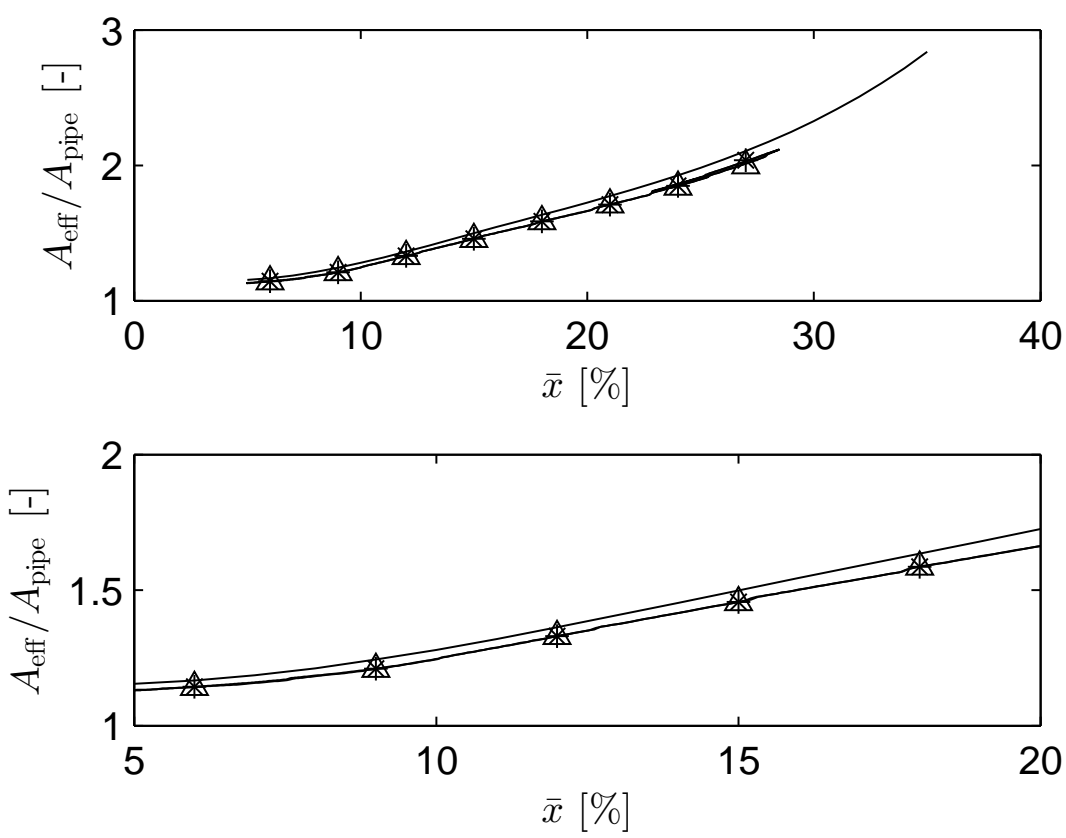

Figure 7. Comparison of the effective areas (-: interpolated steady-state, $\triangle$ : sudden pressure jump, *: slow pressure jump)

As the most important modeled parameter is the effective area, it is beneficial to compare the piecewise cubic interpolation of its steady-state result to those from the two deforming mesh simulations. The various effective areas can be seen in Figure 7. The difference between the two deforming mesh cases is negligible, indicating that the rate of change in the pressure had no effect on the effective area in this parameter range. Since the steady-state curve also runs close to the other curves, it is strongly implied that the basic assumption, i.e. the independence of the effective area from the pipe end pressure was correct for the case of exponentially decaying solutions. The steady-state forces are also somewhat higher, which explains the higher equilibrium lifts of the 1D model in Figure 6.

Flutter usually occurs in configurations where long upstream piping is present [2]. Indeed, unstable behaviour was observed at $p_{\text {set }}=3 \mathrm{bar}, L_{\text {pipe }}=38 D_{\text {pipe }}$ and $p_{\mathrm{t}}(t) \equiv 3.2$ bar. The valve responses can be seen in Figure 8 . While the vibration frequencies are similar, there is a significant difference in the amplitudes. Whether this is due to the absence of some damping effect in the one-dimensional model or the overdamping of pressure waves in the CFD code (which is common for solvers based on the finite volume method) requires further studies and is beyond the scope of this paper. 


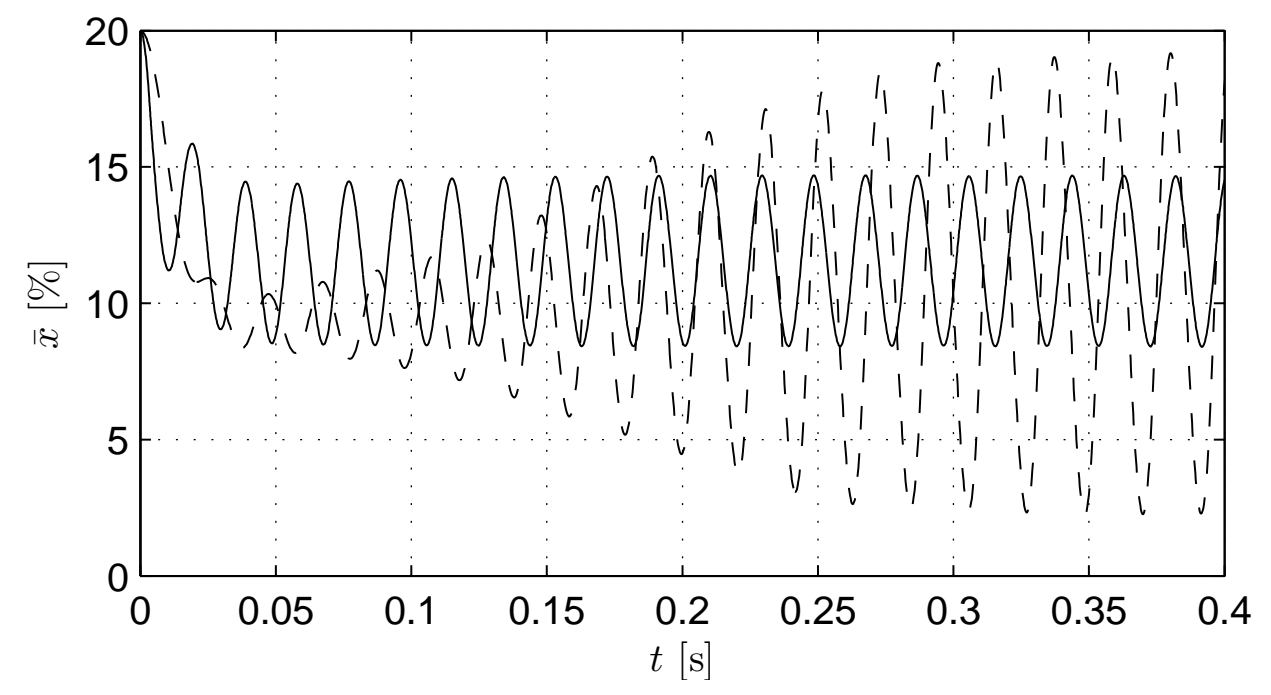

Figure 8. Flutter resolved by the deforming mesh CFD (cont. line) and the 1D (dashed line) models.

\section{Conclusions}

The stationary mesh simulations proved to be computationally cost-effective in mapping a large segment of the $p_{\mathrm{t}}-\bar{x}$ plane both in terms of the effective area and the discharge coefficient. These also made possible the linear stability analysis of various equilibrium solutions. The piecewise cubic interpolation of the effective area and the averaged value of the discharge coefficient were also useful for the $1 \mathrm{D}$ model. The deforming mesh CFD results confirmed that the approximation of the effective area from steady-state flow fields is indeed accurate enough to be used for the modeling of pressure jump scenarios, and that it is also able to qualitatively - and to some extent, quantitatively - produce the same dynamically unstable valve responses as well. Therefore, the 1D model turned out to be an effective and fast tool for transient calculations, albeit strongly depending on the aforementioned input parameters. This behavior also gives flexibility, i.e. it can be easily modified to simulate the response of other PRVs if these parameters are known from CFD simulations or measurements.

Acknowledgement. This research has been supported by the Hungarian Scientific Research Fund OTKA, under grant no. K 116549.

\section{REFERENCES}

1. API: Sizing, Selection, and Installation of Pressure-Relieving Devices in Refineries. API RP520 Part II, Sixth Edition, American Petroleum Institute, Washington, USA, 2013.

2. Hös, C., Champneys, A., Paul, K., and McNeely, M.: Dynamic behavior of direct spring loaded pressure relief valves in gas service: Model development, measurements and instability mechanisms. J. Loss. Prevent. Proc., 31, (2014), 70-81. 
3. BAzsó, C. and Hős, C.: An experimental study on the stability of a direct spring loaded poppet relief valve. J. Fluid. Struct., 42, (2013), 456-465.

4. Zucker, R. D. and Biblarz, O.: Fundamentals of Gas Dynamics. John Wiley \& Sons, 2nd edn., 2002.

5. Ooosthuizen, P. H. and Carscallen, W. E.: Introduction to Compressible Fluid Flow. Taylor \& Francis Group, 2nd edn., 2013.

6. Zucrow, M. J. and Hoffman, J. D.: Gas Dynamics, vol. 1. John Wiley \& Sons, 1976.

7. Blasius, H.: Das Aehnlichkeitsgesetz bei Reibungsvorgängen in Flüssigkeiten. Mitteilungen über Forschungsarbeiten auf dem Gebiete des Ingenieurwesens, 131, (1912), 639-643.

8. Lax, P. and Wendroff, B.: Systems of conservation laws. Commun. Pur. Aappl. Math., 13, (1960), 217-237.

9. Dossena, V., Marinoni, F., Bassi, F., Franchina, N., and Savini, M.: Numerical and experimental investigation on the performance of safety valves operating with different gases. Int. J. Pres. Ves. Pip., 104, (2013), 21-29.

10. Dempster, W. and Elmayyah, W.: Two phase discharge flow prediction in safety valves. Int. J. Pres. Ves. Pip., 110, (2013), 61-65.

11. Song, X.-G., PARK, Y.-C., and PARK, J.-H.: Blowdown prediction of a conventional pressure relief valve with a simplified dynamic model. Math. Comput. Model., 57, (2013), 279-288.

12. Beune, A., Kuerten, J., and van Heumen, M.: CFD analysis with fluid-structure interaction of opening high-pressure safety valves. Comput. Fluids., 64, (2012), 108-116.

13. ANSYS: ANSYS CFX-Solver Theory Guide. Release 14.5, 2012.

14. ANSYS: ANSYS CFX Reference Guide. Release 14.5, 2012.

15. Song, X., Cui, L., Cao, M., CaO, W., Park, Y., and Dempster, W. M.: A CFD analysis of the dynamics of a direct-operated safety relief valve mounted on a pressure vessel. Energ. Convers. Manage., 81, (2014), 407-419. 